\section{SOME PROBLEMS OF REHABILITATION}

\section{BY}

HAROLD BALME, O.B.E., M.D., F.R.C.S.

Emergency Medical Service

Rehabilitation is the popular term of the moment, as freely discussed in non-medical as in medical circles. As is usually. the case with new forms of therapy, there is for the time being a violent swing of the pendulum, and rehabilitation threatens to displace the old-time tonic as a convalescent cure for one and all. This may possibly be followed by an equally violent swing in the opposite direction. But there is little doubt that rehabilitation has come to stay, and will form an integral part of the health services of the future. It is therefore of the utmost importance that its problems should be carefully studied and understood by the profession, and the fullest advantage taken of the present opportunity, created by war conditions, to test out experimental methods and lay the foundations of a sound and scientific scheme.

Nothing would be more unfortunate than for rehabilitation to be divorced from medical or surgical control and established as a new health cult. But that is exactly what may happen unless the profession takes the matter seriously and exercises a guiding and controlling hand; for rehabilitation is a subject which goes far wide of medicine and surgery. Already it is interesting the Ministries of Labour and of Fuel as a means of expediting the recovery of workmen after serious illness or injury and ensuring a more rapid return to employment. It is interesting all who are concerned with physical culture and with the maintenance of general fitness. And not only does it form the central theme of the Tomlinson report but it is written deep in every scheme of post-war sociological planning. There is a real risk of rehabilitation becoming an entity in itself, with socalled rehabilitation centres opened up all over the country without any adequate medical supervision, and a fruitful and most promising form of therapy thus led away along unscientific lines. That will not happen if the medical profession takes firm control from the outset, but to assume such control it is necessary to have a clear view of the problems involved and of the methods by which they may best be solved. It is the purpose of this article to marshal the chief facts connected with rehabilitation, and to attempt to suggest the lines along which healthy development may follow.

In the first place, what do we mean exactly by rehabilitation? It is an unfortunate and clumsy term, and one which is already being used with a variety of different meanings, but from a strictly medical and surgical aspect it can perhaps best be defined as the method by which physiological function is fully restored after its temporary loss from injury or illness. This applies to every form of debilitating disease, accident, or surgical operation, and equally in the physical and the psychological spheres, and no rehabilitation is complete unless it pays due regard to both.

At this point we are met with the objection, already articulate in some quarters, that the physician's task is to cure disease, and that if we will stick to our job Nature will do the rest. We seem to have met that objection before, but it really will not bear a moment's consideration, for the whole purpose of the physician and surgeon, when it comes to questions of ill-health and injury, is to supply what Nature lacks. Every surgical operation, every modern therapeutic agent, every use which we make of serology and roentgenology and physical medicine, bears witness to our conviction that Nature, unaided, will not by any means necessarily perfect her cures. This is as true of the restoration of function as it is of the overcoming of infection, the setting of a fracture, or the removal of a malignant growth. It is therefore incumbent upon us to make a careful study of the factors which prejudice full and speedy return to vigorous health, and the methods by which those obstacles can best be met and overcome.

Let us consider, in the first place, the facts which we require to know as a preliminary to formulating a sound and scientific scheme of rehabilitation. We shall then be in a position to discuss such important questions as the organization of a rehabilitation team, the training of staff, the grading of patients, the provision of the essential facilities, the preparation of handbooks, the relationship of in-patient, out-patient, and posthospital rehabilitation, and the resettlement of the rehabilitated patient in some form of useful employment.

\section{Problems for Research}

A vast amount of time and thought is constantly being expended on the diagnosis and treatment of disease and injury, but surprisingly little attention has ever been paid to the equally important question of the restoration of function. There are innumerable points upon which more research and statistical study is needed before a sound programme of rehabilitation can be drawn up. Here are a few of them:

1. The effect of trauma on every type of soft tissue-fascia, muscle, tendon, serous and synovial membrane, etc.- demands careful study, and we need to know far more accurately than we do at present at what stage, and to what extent, restricted movement is beneficial or harmful, both to healing of the injured part and to the rapid and complete restoration of function. For lack of this exact knowledge many of our rehabilitation methods are of the nature of trial and error-hence the contrary views of those who advocate and those who oppose early remedial exercise. It should not, however, be difficult, by means of controlled experiment carried out on a sufficient number of cases, to establish definite criteria by which to judge of the methods suited to each class of disability and to each stage of recovery.

2. The relationship of pain to immobility is by no means clearly defined; still less do we know of the conditions under which it is wise to abolish pain and promote mobility in diseased or traumatized tissue. It is commonly claimed that pain is Nature's call of aiarm, demanding rest and immobilization for the injured part; but that is by no means the whole of the story. We are all familiar with the type of pain produced by stretched ligaments and adherent soft tissues in which mobilization, and not immobilization, will alone cure both the stiffness and the pain. We are familiar, too, with the so-called "vicious circle of pain," as illustrated by certain injuries in the neighbourhood of joints, the trauma producing effusion which causes pain, and the resultant pain leading to sympathetic disturbance and vasodilatation, and hence to more effusion. In such conditions the abolition of pain by deep novocain injection, as advocated by Leriche, or by intensive freezing with ethyl chloride, certainly hastens the restoration of function in a large number of cases. The same may well be true of other analogous conditions in which pain is associated with immobility. In this connexion an interesting suggestion has been made by a recent colleague of mine, Mr. J. M. Fitton, that the frequency of persistent pain after severe spinal injuries-a frequency much more pronounced than after equally severe injuries in other parts of the body-may not be merely a " litigation neurosis," as so commonly alleged, but may be due to the fibrosis and adhesion of soft tissues, and that where manipulation fails to effect a cure it would be worth while to try the effect of mobilizing the parts by open operation, followed by a course of remedial exercises. It is a suggestion well worth putting to the test.

3 . It is now generally accepted that prolonged immobilization is to be deprecated in the treatment of fractures, owing to its tendency to produce muscle-wasting, stiff and painful joints, and decalcification of bone. What is not so clear, however, is the best time for starting muscular activity. One school of orthopaedic thought states positively that any movement prior to bony union is harmful, and that, provided a well-fitting skin-tight plaster is applied, bony union within reasonable time is certain, after which movement should be started; whereas, according to these authorities, a padded plaster and early movement involve a serious risk of non-union. This view is countered by others who claim that, provided the fracture is in good alignment and controlled by a properly applied padded plaster, changed at intervals, muscular drill within the plaster combined with regular exercise of the more distal joints (e.g., the hip and toes in the case of fracture of the tibia) not only never leads to non-union but actually expedites the date at which consolidation occurs. This is a matter which can only be settled by experiment over a long series of cases, but it is obvious that if the latter contention is the correct one, and the adoption of such measures reduces the period of inactivity of all fracture cases by a week or a fortnight, the resultant gain all over the country, as expressed in man-day power, would be a great economic asset.

4. Too little is yet known as to the factors which hinder return to full use of a joint subjected to open operation, such as a knee-joint after meniscectomy. Most orthopaedic surgeons have to admit to a small minority of cases in which complete recovery is delayed by pain, by lack of full flexion, or by recurrent effusion. How far is this due to inadequate haemostasis at the time of operation; to particular methods of technique; to low-grade sepsis or local irritation; to too early or to too late movement; to excessive or to inadequate quadriceps drill; to too drastic or to too premature attempts at flexion; or to the use of such resistance exercises as those 
provided by weights and pulleys? Far more evidence is needed than is yet available on all these points before we can answer any of these questions, but such evidence is essential if the right methods of rehabilitation are to be chosen for such cases, and-what is perhaps even more important-if patients on whom remedial exercises will have to be slowed up are to be recognized early.

5. The optimum range of movement for every type of disorder, adapted to the stage of recovery, needs to be carefully worked out and formulated. Too often this is left entirely to masseuses or physical instructors, with no medical supervision and no means of checking whether the exercises given are suited to the particular joints or muscles affected, or are too strenuous or not sufficiently active.

6. The remedial exercises in current use have mostly been designed for traumatic cases. But rehabilitation is required for every form of debilitating illness or disorder, not merely for those resulting from accident. Similar care is therefore needed in working out progressive courses of rehabilitation for every variety of medical and surgical complaint. Special exercises are required for thoracic patients, both to promote the activity of the thoracic muscles and to assist good breathing; for post-operative abdominal and hernia cases ; to say nothing of the special needs of head injuries, neurotic and psychotic patients, tuberculous patients, infirm children, etc. There is a great deal of work to be done for each of these groups.

7. The particular apparatus to be employed in the work of rehabilitation, especially the place to be allotted to the use of weights and pulleys; the association of occupational therapy, and its application to the various forms of remedial exercise; the choice of suitable indoor and outdoor recreations and handicrafts, adapted to each type of disability; the place of fatigue in the exercising of traumatized or wasted muscles-all these are examples of other matters requiring careful observation and statistical study.

War conditions have given emergency and Service hospitals a unique opportunity of working out rehabilitation experiments, owing to the fact that in certain of them it is possible to keep patients from the day of injury until they are fit to return to full duty. If each of these centres would concentrate on one of the above problems for research valuable material would be secured on which to base future programmes.

\section{The Grading of Patients}

If remedial exercises and other forms of rehabilitation therapy are to be adapted to the stage of recovery it is essential that some form of grading should be introduced and patients regularly allotted to the particular group to which they belong at any particular time. In one emergency hospital in the North of England to which a large rehabilitation department is attached such a system has been in operation for the past year and has proved of great benefit, both to the patients and to the staff. At this particular hospital five grades are recognized, the programme for each of which is as follows:

Grade 1: Bed patients.-Muscular drill. Faradism and massage where indicated. Assisted exercises. Light occupational therapy, mainly of a diversionary character.

Grade 2: Patients just beginning to get up.-Light remedial exercises. Mild team exercises, of short duration, for patients suffering from similar disabilities and in the same grade. Some remedial occupational therapy, where applicable.

Grade 3.-Stronger exercises, with introduction of some mild form of resistance (e.g., weights and pulleys). Cycling for patients with weakened thigh and leg muscles. Remedial occupational therapy. Organized games, carefully chosen in relation to the particular muscles requiring strengthening or the joints needing to be mobilized.

Grade 4.-Increased resistance introduced into all forms of exercise, both individual and team. Longer periods, including some marching. Heavier occupational therapy (e.g., wood-sawing, digging, carpentry, loom work against resistance springs). More strenuous games.

Grade 5.-A hardening course, including cross-country running, jumping, heavy occupational therapy (e.g., wood-splitting, stoneshifting, etc.), and various team games.

One of the chief values attaching to such a system of grading is that every patient is kept strictly under medical supervision, only the doctor having authority to "up-grade" a patient, or to "de-grade" anyone who is reacting unfavourably to the type of course assigned to his particular group.

\section{Organization of a Rehabilitation Team}

A full rehabilitation team comprises four distinct groups, which may be briefly described as prescribers, medical supervisors, co-ordinating officers, and technicians. In a small hospital, where patients undergoing rehabilitation are but few, these four groups would be reduced to three or even two; but in larger centres all four will be needed, and the closer the co-operation between them the more effective will be their work.

The group entitled "prescribers" will of course consist of the physicians and surgeons actually in charge of the patients, whose duty it is to signify when rehabilitation should start. In some instances they will prefer to supervise this treatment themselves, but in most cases it will be found far better for the work of medical supervision to be delegated to one or more members of the whole-time medical staff, whose duty it will be to keep an eye on the type of exercise, etc., given to each grade, to assist in the training of the technical staff, to supervise the keeping of accurate progress records and individual graphs, and to examine every patient whose improvement is held up by pain or other unfavourable reactions. Such a supervisor will usually be charged with the responsibility of changing patients' grades, as called for, unless the original prescriber prefers to do this himself.

The task of the "co-ordinating officer" is to see that the various forms of remedial exercise, occupational therapy, or recreational therapy laid down for each grade are correctly given ; to arrange the programme of each patient ; to note all new prescriptions ordered by the prescriber or medical supervisor, and see that they are put into force; and, generally speaking, to act as liaison officer between the masseuses, physical training instructors, and occupational therapists, welding them into one unified team of rehabilitation workers.

The practice which has been adopted in some hospitals of holding weekly meetings of the whole team, doctors included, at each of which some particular disability is fully discussed, cannot be too highly praised. At such meetings a preliminary talk is usually given on the anatomy of the particular region under consideration and the diseases or injuries likely to interfere with its function; $x$-ray plates and actual clinical cases are demonstrated; and a free discussion follows as to the best form of physiotherapy, remedial exercises, games, and occupational therapy suitable at each stage of progress.

\section{Training of the Rehabilitation Staff}

This is another problem demanding careful consideration and planning. Rehabilitation should not be regarded as a combined form of therapy to which masseuses, electrotherapists, physical training experts, and occupational therapists each make their own individual and separate contributions. It is essentially a single process, and should be so conceived. Ideally, therefore. the staff should be trained together in an institution attached to a large rehabilitation centre, and this is probably what will happen after the war. For the present this hardly seems practicable, and the various schools of physiotherapy, occupational therapy, and physical culture are therefore attempting to organize courses in active remedial therapy in addition to those which they have previously been offering. This is certainly a move in the right direction, but it still leaves much to be desired, as each section continues to receive training as a separate unit instead of as part of a single rehabilitation team. Possibly the only way to counteract this tendency during wartime will be by the establishment of special postgraduate classes at the larger and better-equipped rehabilitation centres, which masseuses, physical training instructors, and occupational therapists will all be eligible to attend, and which will qualify them for appointment as recognized rehabilitation technicians.

A very urgent need in connexion with the question of training is the provision of suitable textbooks. A comprehensive volume, describing in detail the various forms of remedial exercise. crganized games, and occupational therapy in connexion with rehabilitation, has already been written and should be published in the early autumn; and a valuable handbook on the special remedial exercises suited to each type of disability and to each grade of recovery is now in preparation.

\section{Provision of Essential Facilities}

In considering the problem of how to secure the necessary accommodation, apparatus, and trained staff for the carrying out of rehabilitation it must be remembered that, although the war has provided a unique opportunity of undertaking the whole process of rehabilitation at a single centre, as at certain Service 
and emergency hospitals, this is but a temporary stroke of fortune. Normally, rehabilitation will more often be carried out in three stages, though this must never be allowed to interfere with the continuity of treatment. These stages are:

(a) The period of in-patient treatment, during which the type of rehabilitation therapy described above as Grades 1 and 2 would usually be given.

(b) Out-patient treatment following on discharge from the wards. -Where practicable-e.g., where patients are able to attend daily, and to remain not less than half a day for the purpose of remedial exercises, organized remedial games, and occupational therapy-this would ensure the continuity of rehabilitation in the case of patients who can no longer be retained in the wards and for whom suitable accommodation cannot readily be secured in rehabilitation centres. Such cases might be carried through Grades 2 and 3 without involving the hospital in the necessity for providing very elaborate accommodation or equipment.

(c) Treatment in a special rehabilitation centre.-Such centres will doubtless be set up after the war-in some cases even during the war-in suitable country surroundings, easily accessible from the towns from which patients are received, and equipped with gymnasium, playing fields, occupational therapy workshops, swimming pool, and other requirements for full rehabilitation therapy at all grades. Such centres should of course be intimately connected with the hospital or hospitals from which patients are received, the patients remaining under constant medical supervision, preferably carried out by the supervisor who initiated their rehabilitation treatment in the hospital at which they were first treated.

It will be seen that under such a scheme as the above, ordinary hospitals which do not carry patients beyond Grade 3 will not need to provide extensive playing fields or occupational workshops. What they will need, however, and what they should set about providing at once, are :

(a) A frained staff, keenly interested in active remedial therapy (as opposed to massage, passive movements, radiant heat, etc.) and thoroughly versed in suitable and progressive courses of remedial exercises, games, and occupational therapy.

(b) At least one or two masseuses, and, where possible, a physical training instructor and occupational therapist, trained in remedial work, whose whole time is devoted to active rehabilitation.

(c) A commodious room adapted for use as a gymnasium, remedial exercises, and indoor remedial games, and fitted up with simple apparatus such as weight-and-pulley circuits, wall bars, pronation and supination machine, wrist rollers, ropes, plinths, and benches.

(d) Facilities for providing simple occupational therapy, especially such as is useful for mobilizing stiff fingers, such as basket-work, netting, cane-work, and simple embroidery.

(e) A suitable place within or adjoining the hospital grounds for such organized outdoor games as medicine ball, netball, etc.

It must be remembered that, to be thoroughly successful, rehabilitation should occupy best part of a patient's day. It is not enough to arrange for attendance at a course of exercises for a brief period three or four times a week, or even daily, if the rest of the day is left unoccupied. One of the greatest values of rehabilitation is on the psychological side, as the patient's mind gets directed towards his return to full health and he is encouraged to co-operate in the process. For that reason his day should be filled up as fully as possible, not merely with courses of teain exercises-his muscles will soon tire if such exercises are continued beyond a half-hour or forty-minute period-but also with other forms of exercise, games, and remedial handicrafts. Very few hospitals yet have the accommodation or staff to provide such facilities for a whole day for any one patient; but all should aim at a full half-day programme for each patient, some attending for morning sessions and the remainder in the afternoon or early evening.

\section{Resettlement in Industry}

The task of resettling convalescent patients in employment is obviously not one which devolves primarily on the medical profession, but if the work of rehabilitation is to be carried to its logical and desirable conclusion the collaboration of hospital authorities and of medical supervisors will be essential. This will take the form of advising when a particular patient is fit to return to duty; of indicating whether he can return to his former employment or should be given lighter duties, or, alternatively, whether he should be trained for some different and more suitable vocation; of establishing relations with industrial medical officers in the carrying out of such recommendations; and, where possible, of reviewing the case after return to full work, either by means of medical reports or by actual examination of the patient himself.

In carrying out this essential terminal phase of rehabilitation the assistance of specially trained social workers, attached to hospitals and rehabilitation centres, is of the utmost importance. It is they who should keep track of each patient's attendance at the rehabilitation department, looking up any who are persistently absent. They should receive the medical officer's recommendations as to patients' return to duty. They should keep in intimate touch with employers and arrange for the reengagement of those fit to start work. They should make contact with the Labour Exchange authorities in relation to cases that require to be trained in one of the vocational training centres established by the Ministry of Labour. Finally, they should be qualified to assist the patient in the intricate problems arising out of the present laws governing workmen's compensation, encouraging him to attempt light duties in spite of the reduction in disability allowance, while at the same time keeping industrial medical officers informed of the condition of each worker returning to work in factory or mine after illness or injury.

\section{Summary}

Rehabilitation is defined as the method by which physiological and psychological function are fully restored after their temporary loss from injury or illness.

An organized system of rehabilitation should be regarded as an integral part of the treatment of all debilitating diseases and injuries.

It is essential that it should not be divorced from medical and surgical control, but such dissociation is liable to occur unless its problems are closely studied by the profession and a sound scheme is adopted by all hospital authorities.

As a guide to the formulation of such a scheme a list is suggested of subjects on which further research and statistical study are required.

A system by which patients undergoing rehabilitation are graded according to their stage of recovery is recommended.

The various groups which should constitute a rehabilitation team, and the responsibility devolving on each, are outlined.

The training of the rehabilitation staff, and the provision of the facilities required by hospitals and by special rehabilitation centres, are discussed.

The contribution of rehabilitation medical officers and of hospital social workers towards the resettlement of patients in industry is indicated.

\section{Nova et Vetera}

\section{TEACHING OF ANATOMY}

Mr. CONNOR BANTRY White of Westminster Hospital Medical School writes: The following extract, taken from an old anatomy book in my possession, shows that the concern felt by teachers of anatomy is by no means recent, and, I think, aptly summarizes the opinions put forward by your correspondents: "The study of Anatomy, as it leads to the knowledge of nature and the art of healing, needs not many tedious descriptions nor minute dissections; what is most worth knowing is soonest learned, and least the subject of disputes; while dividing and describing the parts, more than the knowledge of their uses requires, perplexes the learner and makes the science dry and difficult." This is the first sentence of the preface to the ninth edition of The Anatomy of the Human Body, by W. Cheselden, Surgeon to His Majesty's Royal Hospital at Chelsea. His book was printed in London in 1768.

After several refusals to go to the U.S.A., von Helmholtz was persuaded by the German Government to represent German science at the Electrical Congress held in Chicago in 1893. In a letter to Dr. Knapp, announcing the date of his arrival in New York, von Helmholtz, who was then 71, wrote: "I am convinced that America represents the future of civilized humanity and that it includes a vast number of interesting people, while in Europe we see only the approach of chaos or of a world dominated by Russia." Dr. Knapp, who was the recipient of this letter, was the founder of the Archives of Ophthalmology and the father of the present chief editor of that journal. ("Purkyne, a Pioneer in Ophthalmoscopy." William Thau. Arch. Ophthal., Chicago, 1942, 27, 299.) 